\title{
LAPLACE TRANSFORMS AND GENERALIZED LAGUERRE POLYNOMIALS
}

\author{
P. G. ROONEY
}

1. Introduction. Various sets of necessary and sufficient conditions are known in order that a function $f(s)$, analytic for $\operatorname{Re} s>0$, be represented as the Laplace transform of a function in $L_{p}(0, \infty), 1<p \leqslant \infty$. Most of these theories are based on the properties of some inversion operator for the transformation-see, for example, (7, chap. 7). However in the case $p=2$ a number of representation theorems of a much simpler type are available. One of these is due to Shohat (5) who has in effect shown that a necessary and sufficient condition for such a representation, with $p=2$, is that

$$
\sum_{n=0}^{\infty}\left|q_{n}\right|^{2}<\infty
$$

where

$$
q_{n}=\sum_{r=0}^{n}\left(\begin{array}{c}
n \\
r
\end{array}\right) \frac{1}{r !} f^{(r)}\left(\frac{1}{2}\right) .
$$

Shohat's proof makes use of the Laguerre polynomials.

Recently the author has given (4) necessary and sufficient conditions that $f(s)$ be the Laplace transform of a function of the form $t^{\lambda} F(t), F \in L_{p}(0, \infty)$, $1<p \leqslant \infty, \lambda>-1 / q$, where $p^{-1}+q^{-1}=1$. These conditions were given in terms of a particular inversion operator. In this paper we shall see that Shohat's theorem can be generalized, for $p=2$, to cover this more general case. This is done in $\S 2$ below, using generalized Laguerre polynomials. We also obtain there an expression for $F(t)$ which we shall use in $\S 3$ to obtain some results about Hankel transforms. For convenience we write $\lambda=\frac{1}{2} \nu$ throughout the following.

2. Representation theorem. We start with a preliminary lemma.

LEMMA 1. If $f(s)$ is analytic for $\operatorname{Re} s>0$ and $\nu>-1$, then

$$
f(s)=\frac{1}{\left(s+\frac{1}{2}\right)^{\nu+1}} \sum_{n=0}^{\infty} q_{n}\left(\frac{s-\frac{1}{2}}{s+\frac{1}{2}}\right)^{n},
$$

where

$$
q_{n}=\sum_{r=0}^{n}\left(\begin{array}{l}
n+\nu \\
n-r
\end{array}\right) \frac{1}{r !} f^{(r)}\left(\frac{1}{2}\right),
$$

the branch of $\left(s+\frac{1}{2}\right)^{\nu+1}$ that is positive when $s+\frac{1}{2}$ is positive being chosen.

Received May 31, 1957. This work was done in part while the author was the holder of a summer associateship of the National Research Council of Canada. 
Proof. Let

$$
s=\frac{1}{2} \frac{1+z}{1-z},
$$

and $f(s)=F(z)$. Then $F(z)$ is analytic in $|z|<1$, and hence so is $F(z) /(1-z)^{\nu+1}$. Thus

$$
F(z) /(1-z)^{\nu+1}=\sum_{n=0}^{\infty} q_{n} z^{n}, \quad|z|<1,
$$

where if $r<1$

$$
\begin{aligned}
q_{n} & =\frac{1}{2 \pi i} \int_{|z|=r}\left(F(z) / z^{n+1}(1-z)^{\nu+1}\right) d z \\
& =\operatorname{Residue~}_{z=0}\left(F(z) / z^{n+1}(1-z)^{\nu+1}\right) \\
& =\operatorname{Residue~}_{s=\frac{1}{2}}\left(f(s)\left(s+\frac{1}{2}\right)^{n+\nu} /\left(s-\frac{1}{2}\right)^{n+1}\right) \\
& =\frac{1}{n !} \lim _{s \rightarrow \frac{1}{2}}\left\{\frac{a^{n}}{d s^{n}}\left(f(s)\left(s+\frac{1}{2}\right)^{n+\nu}\right)\right\} \\
& =\frac{1}{n !} \lim _{s \rightarrow \frac{1}{2}}\left\{\sum_{r=0}^{n}\left(\begin{array}{l}
n \\
r
\end{array}\right) f^{(r)}(s) \frac{\Gamma(n+\nu+1)}{\Gamma(r+\nu+1)}\left(s+\frac{1}{2}\right)^{r+\nu}\right\} \\
& =\sum_{r=0}^{n}\left(\begin{array}{l}
n+\nu \\
n-r
\end{array}\right) \frac{1}{r !} f^{(r)}\left(\frac{1}{2}\right) .
\end{aligned}
$$

Hence

$$
f(s)=F(z)=(1-z)^{\nu+1} \sum_{n=0}^{\infty} q_{n} z^{n}=\frac{1}{\left(s+\frac{1}{2}\right)^{\nu+1}} \sum_{n=0}^{\infty} q_{n}\left(\frac{s-\frac{1}{2}}{s+\frac{1}{2}}\right)^{n} .
$$

TheOREM 1. A necessary and sufficient condition that a function $f(s)$, analytic for $\operatorname{Re} s>0$, be the Laplace transform of a function of the form $t^{\frac{1}{2} \nu} F(t)$, with $F \in L_{2}(0, \infty)$ and $\nu>-1$, is that

$$
\sum_{n=0}^{\infty} \frac{n !}{\Gamma(\nu+n+1)}\left|q_{n}\right|^{2}<\infty
$$

where

$$
q_{n}=\sum_{r=0}^{n}\left(\begin{array}{l}
n+\nu \\
n-r
\end{array}\right) \frac{1}{r !} f^{(r)}\left(\frac{1}{2}\right) .
$$

In this case

$$
F(t)=\underset{r \rightarrow \infty}{\operatorname{li} . m} . t^{\frac{1}{2} \nu} e^{-\frac{1}{2} t} \sum_{n=0}^{r} \frac{n !}{\Gamma(\nu+n+1)} q_{n} L_{n}^{(\nu)}(t),
$$

and

$$
\sum_{n=0}^{\infty} \frac{n !}{\Gamma(\nu+n+1)}\left|q_{n}\right|^{2}=\int_{0}^{\infty}|F(t)|^{2} d t
$$


Proof of necessity. Suppose

$$
f(s)=\int_{0}^{\infty} e^{-s t} t^{\frac{1}{2} \nu} F(t) d t, \quad F \in L_{2}(0, \infty), \nu>-1 .
$$

Let

$$
\phi_{n}(t)=\left(\frac{n !}{\Gamma(\nu+n+1)}\right)^{\frac{1}{2}} e^{-\frac{1}{2} t} t^{\frac{1}{2} \nu} L_{n}^{(\nu)}(t) .
$$

Then, as is well known, $\left\{\phi_{n}\right\}$ is a complete orthonormal sequence in $L_{2}(0, \infty)$. We have, using $(2, \S 10.12(7))$ and $(1$, chap. $3, \S 2)$,

$$
\begin{aligned}
\left(F, \phi_{n}\right) & =\left(\frac{n !}{\Gamma(\nu+n+1)}\right)^{\frac{1}{2}} \int_{0}^{\infty} e^{-\frac{1}{2} t} t^{\frac{1}{2} \nu} L_{n}^{(\nu)}(t) F(t) d t \\
& =\left(\frac{n !}{\Gamma(\nu+n+1)}\right)^{\frac{1}{2}} \sum_{r=0}^{n}\left(\begin{array}{l}
n+\nu \\
n-r
\end{array}\right) \frac{1}{r !} \int_{0}^{\infty} e^{-\frac{1}{2} t}(-t)^{r} t^{\frac{1}{2} \nu} F(t) d t \\
& =\left(\frac{n !}{\Gamma(\nu+n+1)}\right)^{\frac{1}{2}} \sum_{r=0}^{\infty}\left(\begin{array}{l}
n+\nu \\
n-r
\end{array}\right) \frac{1}{r !} f^{(r)}\left(\frac{1}{2}\right)=\left(\frac{n !}{\Gamma(\nu+n+1)}\right)^{\frac{1}{2}} q_{n} .
\end{aligned}
$$

Hence

$$
\begin{aligned}
F(t) & =\underset{r \rightarrow \infty}{\lim . \mathrm{m}} \sum_{n=0}^{r}\left(F, \phi_{n}\right) \phi_{n}(t) \\
& =\underset{r \rightarrow \infty}{\operatorname{1.i.m} .} t^{\frac{1}{2} \nu} e^{-\frac{1}{2} t} \sum_{n=0}^{r} \frac{n !}{\Gamma(\nu+n+1)} q_{n} L_{n}^{(\nu)}(t),
\end{aligned}
$$

and from the Parseval relation

$$
\sum_{n=0}^{\infty} \frac{n !}{\Gamma(\nu+n+1)}\left|q_{n}\right|^{2}=\sum_{n=0}^{\infty}\left|\left(F, \phi_{n}\right)\right|^{2}=\int_{0}^{\infty}|F(t)|^{2} d t<\infty
$$

Proof of sufficiency. Since

$$
\sum_{n=0}^{\infty} \frac{n !}{\Gamma(\nu+n+1)}\left|q_{n}\right|^{2}<\infty
$$

by the Riesz-Fischer theorem there is a function $F \in L_{2}(0, \infty)$ such that

$$
\left(F, \phi_{n}\right)=q_{n}\left(\frac{n !}{\Gamma(\nu+n+1)}\right)^{\frac{1}{2}} \text {. }
$$

Let $G(t)=t^{\frac{1}{2} v} e^{-\bar{s} t}, \operatorname{Re} s>0$. Then $G \in L_{2}(0, \infty)$, and from $(3, \S 4.11(28))$,

$$
\begin{aligned}
\left(G, \phi_{n}\right) & =\int_{0}^{\infty} t^{\frac{1}{2} \nu} e^{-\bar{s} t} \phi_{n}(t) d t \\
& =\left(\frac{n !}{\Gamma(\nu+n+1)}\right)^{\frac{1}{2}} \int_{0}^{\infty} e^{-\left(\bar{s}+\frac{1}{2}\right) t} t^{\nu} L_{n}^{(\nu)}(t) d t \\
& =\left(\frac{\Gamma(\nu+n+1)}{n !}\right)^{\frac{1}{2}} \frac{\left(\bar{s}-\frac{1}{2}\right)^{n}}{\left(\bar{s}+\frac{1}{2}\right)^{n+\nu+1}} .
\end{aligned}
$$


Hence from Lemma 1 and Parseval's relation, if $\operatorname{Re} s>0$,

$$
\begin{aligned}
f(s) & =\frac{1}{\left(s+\frac{1}{2}\right)^{\nu+1}} \sum_{r=0}^{\infty} q_{n}\left(\frac{s-\frac{1}{2}}{s+\frac{1}{2}}\right)^{n} \\
& =\sum_{n=0}^{\infty}\left\{q_{n}\left(\frac{n !}{\Gamma(\nu+n+1)}\right)^{\frac{1}{2}}\right\}\left\{\left(\frac{\Gamma(\nu+n+1)}{n !}\right)^{\frac{1}{2}} \frac{\left(s-\frac{1}{2}\right)^{n}}{\left(s+\frac{1}{2}\right)^{n+\nu+1}}\right\} \\
& =\sum_{n=0}^{\infty}\left(F, \phi_{n}\right) \overline{\left(G, \phi_{n}\right)}=(F, G)=\int_{0}^{\infty} e^{-s t} t^{\frac{1}{2} \nu} F(t) d t .
\end{aligned}
$$

3. Application to Hankel transforms. For our purposes here we shall define the Hankel transform for $F \in L_{2}(0, \infty), \nu>-1$, by

$$
G(x)=\frac{d}{d x} \int_{0}^{\infty} k_{\nu}(x y) F(y) \frac{d y}{y}
$$

where

$$
k_{\nu}(x)=\int_{0}^{x} J_{\nu}(2 \sqrt{ } y) d y .
$$

Since the Mellin transform of $J_{\nu}(2 \sqrt{ } y)$ is

$$
\Gamma\left(s+\frac{1}{2} \nu\right) / \Gamma\left(\frac{1}{2} \nu-s+1\right), \quad-\frac{1}{2} \nu<\operatorname{Re} s<3 / 4,
$$

it follows since $\nu>-1$ that the hypotheses of (6, Theorem 129) are satisfied so that if $F \in L_{2}(0, \infty), G$ exists and is in $L_{2}(0, \infty)$, and Parseval's equation holds. Further

$$
F(x)=\frac{d}{d x} \int_{0}^{\infty} k_{\nu}(x y) G(y) \frac{d y}{y} .
$$

Here we shall use the results of Theorem 1 to invert the Hankel transform. We first prove the following lemma (compare (1, chap $2 \S 16)$ ).

Lemma 2. If $F \in L_{2}(0, \infty), \nu>-1$,

$$
G(x)=\frac{d}{d x} \int_{0}^{\infty} k_{\nu}(x y) F(y) d y
$$

where

$$
\begin{aligned}
k_{\nu}(x) & =\int_{0}^{x} J_{\nu}(2 \sqrt{ } y) d y, \\
f(s) & =\int_{0}^{\infty} e^{-s t} t^{\frac{1}{\nu}} F(t) d t
\end{aligned}
$$

and

$$
g(s)=\int_{0}^{\infty} e^{-s t} t^{\frac{1}{2} \nu} G(t) d t,
$$

then

$$
f(s)=\frac{1}{s^{\nu+1}} g(1 / s) .
$$


Proof. The Hankel transform of $t^{\frac{1}{2} v} e^{-s t}$ is given, on using $(3, \S 4.14(30))$, by

$$
\begin{aligned}
\frac{d}{d x} \int_{0}^{\infty} t^{\frac{1}{2} \nu-1} e^{-s t} d t \int_{0}^{x t} J_{\nu}(2 \sqrt{ } y) d y \\
\quad=\frac{d}{d x} \int_{0}^{\infty} t^{\frac{1}{2} \nu} e^{-s t} d t \int_{0}^{x} J_{\nu}(2 \sqrt{ } y t) d y \\
\quad=\frac{d}{d x} \int_{0}^{x} d y \int_{0}^{\infty} t^{\frac{1}{2} \nu} e^{-s t} J_{\nu}(2 \sqrt{ } y t) d t \\
\quad=\int_{0}^{\infty} t^{\frac{1}{2} \nu} e^{-s t} J_{\nu}(2 \sqrt{ } x t) d t \\
=\frac{x^{\frac{1}{2} \nu} e^{-x / s}}{s^{\nu+1}},
\end{aligned}
$$

the interchange of the order of integrations being justified by Fubini's theorem.

Hence by the Parseval relation for the Hankel transform,

$$
\begin{aligned}
f(s) & =\int_{0}^{\infty} e^{-s t} t^{\frac{1}{2} \nu} F(t) d t=\frac{1}{s^{\nu+1}} \int_{0}^{\infty} e^{-t / s} t^{\frac{1}{2} \nu} G(t) d t \\
& =\frac{1}{s^{\nu+1}} g\left(\frac{1}{s}\right) .
\end{aligned}
$$

Theorem 2. If $F \in L_{2}(0, \infty), \nu>-1$,

$$
G(x)=\frac{d}{d x} \int_{0}^{\infty} k_{\nu}(x y) F(y) \frac{d y}{y}
$$

where

$$
k_{\nu}(x)=\int_{0}^{x} J_{\nu}(2 \sqrt{ } y) d y
$$

and

$$
g(s)=\int_{0}^{\infty} e^{-s t} t^{\frac{1}{2} \nu} G(t) d t
$$

then

$$
F(t)=\underset{r \rightarrow \infty}{\operatorname{lix} . \mathrm{m}} t^{\frac{1}{2} \nu} e^{-\frac{1}{2} t} \sum_{n=0}^{r} q_{n} \frac{n !}{\Gamma(\nu+n+1)} L_{n}^{(\nu)}(t)
$$

where

$$
q_{n}=(-1)^{n} 2^{\nu+1} \sum_{r=0}^{n}\left(\begin{array}{l}
n+\nu \\
n-r
\end{array}\right) \frac{4^{r}}{r !} g^{(r)}(2) .
$$

Proof. By Theorem 1,

$$
F(t)=\underset{r \rightarrow \infty}{\operatorname{li} . \mathrm{m} .} t^{\frac{1}{2} \nu} e^{-\frac{1}{2} t} \sum_{n=0}^{r} q_{n} \frac{n !}{\Gamma(\nu+n+1)} L_{n}^{(\nu)}(t)
$$

where

$$
q_{n}=\sum_{r=0}^{n}\left(\begin{array}{l}
n+\nu \\
n-r
\end{array}\right) \frac{1}{r !} f^{(r)}\left(\frac{1}{2}\right)
$$


But in the proof of Lemma 1 we showed that

$$
q_{n}=\operatorname{Residue}_{s=\frac{1}{2}}\left(f(s)\left(s+\frac{1}{2}\right)^{n+\nu} /\left(s-\frac{1}{2}\right)^{n+1}\right),
$$

and hence using Lemma 2

$$
\begin{aligned}
q_{n} & =\text { Residue }_{s=\frac{1}{2}}\left(\frac{1}{s^{\nu+1}} g\left(\frac{1}{s}\right)\left(s+\frac{1}{2}\right)^{n+\nu} /\left(s-\frac{1}{2}\right)^{n+1}\right) \\
& =\text { Residue }_{s=2} \frac{-1}{2^{\nu-1}}\left(g(s)(2+s)^{n+\nu} /(2-s)^{n+1}\right) \\
& =\frac{(-1)^{n}}{2^{\nu-1} n !} \lim _{s \rightarrow 2} \frac{d^{n}}{d s^{n}}\left(g(s)(2+s)^{n+\nu}\right) \\
& =\frac{(-1)^{n}}{2^{\nu-1} n !} \lim _{s \rightarrow 2} \sum_{r=0}^{n}\left(\begin{array}{c}
n \\
r
\end{array}\right) g^{(r)}(s) \frac{\Gamma(n+\nu+1)}{\Gamma(r+\nu+1)}(s+2)^{r+\nu} \\
& =(-1)^{n} 2^{\nu+1} \sum_{r=0}^{n}\left(\begin{array}{l}
n+\nu \\
n-r
\end{array}\right) \frac{4^{r}}{r !} g^{(r)}(2) .
\end{aligned}
$$

Corollary. Under the hypotheses of Theorem 2, if

$$
f(s)=\int_{0}^{\infty} e^{-s t} t^{\frac{1}{2} \nu} F(t) d t
$$

then

$$
G(t)=\underset{r \rightarrow \infty}{\lim . \mathrm{m}} t^{\frac{1}{2} \nu} e^{-\frac{1}{2} t} \sum_{n=0}^{r} q_{n}^{\prime} \frac{n !}{\Gamma(\nu+n+1)} L_{n}^{(\nu)}(t)
$$

where

$$
q_{n}^{\prime}=(-1)^{n} 2^{\nu+1} \sum_{r=0}^{n}\left(\begin{array}{l}
n+\nu \\
n-r
\end{array}\right) \frac{4^{\tau}}{r !} f^{(r)}(2)
$$

Proof. This follows from Theorem 2 since the relation between $F$ and $G$ is reciprocal.

\section{REFERENCES}

1. G. Doetsch, Handbuch der Laplace Transformation I (Basel, 1950).

2. A. Erdélyi et al., Higher Transcendental Functions II (New York, 1953).

3. A. Erdélyi et al., Tables of Integral Transforms I (New York, 1954).

4. P. G. Rooney, On an inversion formula for the Laplace transformation, Can. J. Math., 7 (1955), 101-115.

5. J. Shohat, Laguerre polynomials and the Laplace transform, Duke Math. J., 6 (1940), 615-626.

6. E. C. Titchmarsh, Introduction to the Theory of Fourier Integrals (Oxford, 1948).

7. D. V. Widder, The Laplace Transform (Princeton, 1941).

\section{University of Toronto}

